\title{
Degradation of Mg-doped zinc oxide buffer layers in thin film CdTe solar cells
}

\author{
Francesco Bittau, Shridhar Jagdale, Christos Potamialis, Jake W. Bowers and John M. Walls \\ Centre for Renewable Energy Systems Technology (CREST), Wolfson School of \\ Mechanical, Electrical and Manufacturing Engineering, Loughborough University, \\ Loughborough, Leicestershire, LE11 3TU, U.K. \\ Amit H. Munshi, Kurt L. Barth and Walajabad S. Sampath \\ Department of Mechanical Engineering, Colorado State University, Fort Collins, CO 80523 \\ U.S.A.
}

\begin{abstract}
Cadmium Sulphide is the conventional n-type buffer layer used in thin film Cadmium Telluride solar cells. It is well known that Cadmium Sulphide causes optical losses and sulphur diffuses into the absorber during high temperature activation. Sputterdeposited Mg-doped ZnO (MZO) has been shown to be an attractive buffer layer for Cadmium Telluride solar cells due to its transparency and tuneable band gap. It is also stable to high temperature processing and avoids diffusion of elements into the cadmium telluride absorber during the cadmium chloride activation treatment. However, degradation is observed in solar cells incorporating MZO buffer layers. Analysis of the MZO film surface potential has revealed significant fluctuations in the thin film work function once the layer is exposed to the atmosphere following deposition. These fluctuations are attributed to the high reactivity to water vapour of the $\mathrm{MgO}$ contained in the $\mathrm{MZO}$ films. This has been analysed using X-ray Photoelectron Spectroscopy to determine corresponding changes in the surface chemistry. The Zinc Oxide component is relatively stable, but the analysis shows that $\mathrm{MgO}$ forms a $\mathrm{Mg}(\mathrm{OH})_{2}$ layer on the $\mathrm{MZO}$ surface which forms a secondary barrier at the $\mathrm{MZO} / \mathrm{CdTe}$ interface and/or at the interface between $\mathrm{MZO}$ and the Fluorine-doped $\mathrm{SnO}_{2}$. This affects the Fill Factor and as a consequence it degrades the conversion efficiency.

KEYWORDS: Magnesium-doped Zinc Oxide, thin film solar cells, Cadmium telluride, buffer, degradation, hydroxide, surface contamination.
\end{abstract}

\section{INTRODUCTION}

Thin film solar cells are heterostructure devices formed using a stack of several different semiconductor, metal-oxide and metallic materials as shown schematically in Fig. 1. Thin film cadmium telluride photovoltaic devices are commercially successful because they are the lowest cost solar technology. Cadmium Telluride ( $\mathrm{CdTe}$ ) is a direct band gap absorber which is typically only 4 um in thickness compared with $\approx 150$ um for crystalline silicon. This provides thin film technology with a natural cost advantage. Cadmium Telluride solar cells are fabricated in the superstrate configuration on glass coated with a transparent conducting oxide (TCO) which serves as the front contact. This TCO is usually Fluorine doped 
tin oxide (FTO). In the conventional cell architecture, a thin Cadmium Sulphide (CdS) n-type buffer layer has been used to form the $p-n$ junction. $\mathrm{CdS}$ has a band gap of $2.45 \mathrm{eV}$ which causes parasitic absorption losses in the blue end of the spectrum. Oxygen can be added to the CdS to form CdS:O to increase the band gap but absorption is not eliminated [1]-[4]. In addition, CdS is not stable at high temperatures and significant diffusion of sulphur into the CdTe absorber occurs during the cadmium chloride activation treatment. Tellurium also diffuses into the CdS [5]. Ideally, the CdS should be replaced by a layer that is highly transparent with a band gap $>3 \mathrm{eV}$ and which also creates an optimal interface with the absorber layer. The transparency of this layer determines the number of photons reaching the absorber and consequently, the number of electron-hole pairs generated, thereby increasing the short circuit current (Jsc). An optimised buffer/absorber interface is essential to maximise the open circuit Voltage(Voc) and Fill Factor (FF) [6]-[8], and the quality of this interface depends on the interface chemistry, the lattice mismatch and the band alignment between the semiconductors. The device is completed with a back contact, deposited on top of the absorbing material, normally consisting of a buffer layer and a metal contact. The back of the cell is usually doped with copper sometimes in the form of ZnTe:Cu.

Mg-doped ZnO (MZO) has been shown to be an effective buffer layer for both CdTe and CdSeTe alloyed cells, due to its high transparency. It is also possible to tune its energy band structure to fit the absorber material [6], [8]-[13]. By adding $\mathrm{Mg}$ to the $\mathrm{ZnO}$ lattice, the substitution of $\mathrm{Zn}$ ions with $\mathrm{Mg}$ to form $\mathrm{MgO}$ causes an upward shift of the conduction band level of the semiconductor. This is because the $\mathrm{MgO}$ band gap $(7.8 \mathrm{eV})$ is much larger than $\mathrm{ZnO}(3.3 \mathrm{eV})$. The use of MZO increases the Jsc and has resulted in near record cell efficiencies [14]. Here we report on a degradation mechanism that explains the change in CdTe efficiency observed once the cells are exposed to the atmosphere. The degradation observed affects all solar cell parameters but with the most significant deterioration affecting the Fill Factor. Although the observations here are made pertaining to the use of MZO in CdTe devices, the degradation is also likely to occur when this material is used in CIGS devices. 


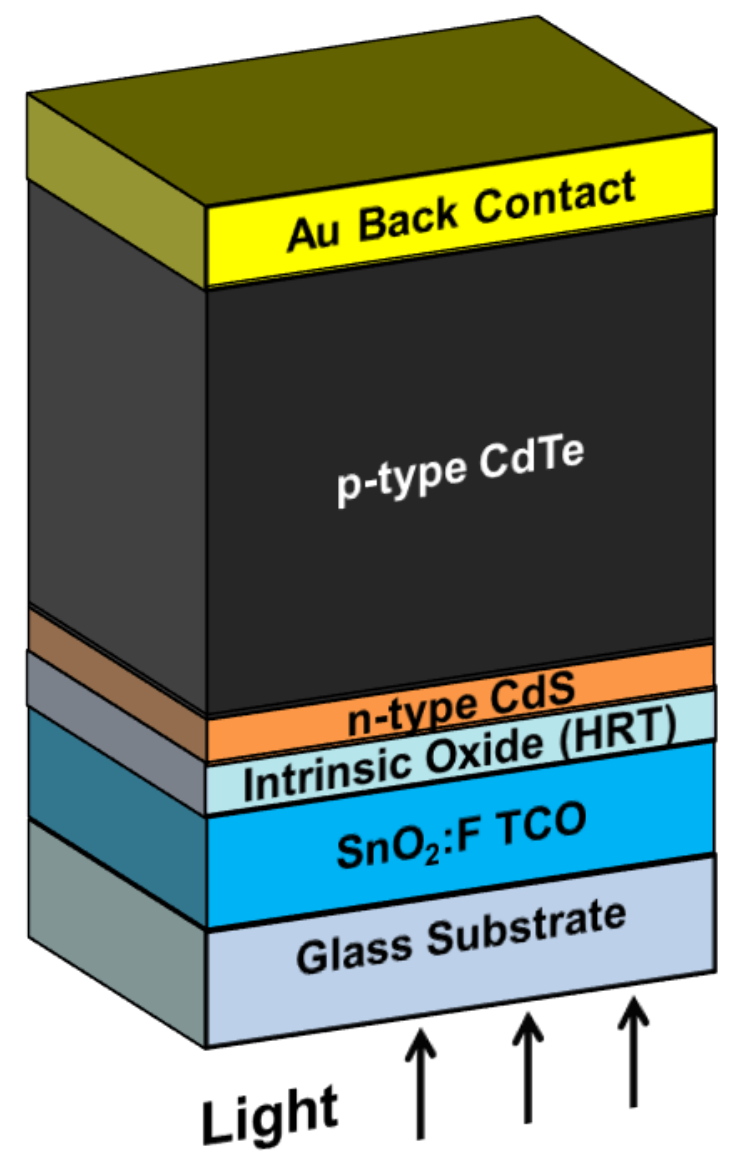

Fig. 1: Schematic diagram of the conventional structure of a thin film CdTe solar device. The use of $\mathrm{CdS}$ causes parasitic absorption losses which can be eliminated by using a wide band gap buffer layer such as magnesium doped zinc oxide (MZO).

\section{EXPERIMENTAL DETAILS}

The MZO samples were deposited onto $1 \mathrm{~mm}$ thick soda lime glass for characterisation or NSG TECTM C10 glass coated with a FTO TCO for device fabrication. The glass substrates were cleaned in a solution of soap in deionised (DI) water for 30 mins in an ultrasonic bath at room temperature. This was followed by cleaning in a $1 / 3$ solution of acetone in an ultrasonic bath for 30 mins at room temperature. Finally, the glass was cleaned in a 1/3 solution of isopropanol in an ultrasonic bath for 90 mins. The thin film of MZO was deposited using an Orion $8 \mathrm{HV}$ magnetron sputtering system (AJA International, USA) equipped with an AJA 600 series radio-frequency (RF) power supply. The compound sputtering target of MZO had a composition of $11 \%$ wt.\% Magnesium oxide (MgO) and $89 \%$ wt.\% Zinc oxide $(\mathrm{ZnO})$ and was 3 " in diameter. The glass substrates were rotated at $10 \mathrm{rpm}$ during deposition to ensure thin film uniformity. The MZO films used for fabrication of CdTe solar cells were prepared at $1 \% \mathrm{O}_{2}$ in Ar working gas, $180 \mathrm{~W}$ power and at ambient temperature, $20^{\circ} \mathrm{C}$. MZO thin film samples were prepared for further characterisation, by changing the reactive working gas composition or the sputtering power. A first batch of samples was prepared at a constant power of $150 \mathrm{~W}$ and by varying the oxygen content 
between $0 \%$ and $2 \%$ in Argon. The second batch of MZO films was prepared by changing the sputtering power with the range between $120 \mathrm{~W}$ and $180 \mathrm{~W}$ and keeping the oxygen levels constant of at $1 \%$. The deposition duration was 2 hours for all samples. The optical properties of the MZO thin films were measured using Varian Cary 5000 UV-VIS-NIR spectrophotometer. The composition of the thin films was measured using a X-ray Photoelectron spectroscopy (Thermo Scientific K-alpha) using Al X-rays. The structural properties of the films were analysed by using a Brucker D2 phaser desktop X-ray diffractometer equipped with a Cu-K-Alpha X-ray gun. The surface potential of the films was measured with an ambient Kelvin probe from KP Technology Ltd. The surface work function was then calculated by using a gold calibration sample, assuming a tip work function of 4800 meV. The ageing process for MZO films and for CdTe devices took place contemporarily inside the same desiccator, to ensure the environmental conditions were identical for all samples throughout. The CdTe absorber was deposited by close space sublimation (CSS) at a pressure of $133.3 \mathrm{~Pa}$ in a $6 \% \mathrm{O}_{2}$ in Ar atmosphere, at a CdTe source plate temperature of $630{ }^{\circ} \mathrm{C}$ and a substrate temperature of $515^{\circ} \mathrm{C}$, for 3 minutes. The spacing between substrate and source plate was set to $2 \mathrm{~mm}$. The $\mathrm{CdCl}_{2}$ activation treatment was carried out by thermal evaporation and subsequent annealing. A quartz crucible was loaded with $0.5 \mathrm{~g}$ of $\mathrm{CdCl} 2$ pellets, which was then evaporated at $\sim 1.33 \times 10-4 \mathrm{~Pa}$ for 20 minutes. The sample was then annealed on a hot plate at dwell temperature of $425^{\circ} \mathrm{C}$ and $1 \mathrm{~min}$ dwell time. The dwell temperature was reached by using a $22 \%$ min ramping rate bringing the temperature from room temperature to the dwell temperature in 18 minutes. Devices were rinsed with DI water to clean the $\mathrm{CdTe}$ surface from $\mathrm{CdCl}_{2}$ residues and completed with $80 \mathrm{~nm}$ gold contacts deposited using thermal evaporation. No intentional copper has been added to these devices to exclude the effect of $\mathrm{Cu}$ diffusion contributing to device degradation.

\section{RESULTS}

3.1. The degradation of CdTe solar cells incorporating a MZO buffer layer

CdTe solar cells were fabricated on glass substrates coated with a FTO TCO and a $100 \mathrm{~nm}$ thick $\mathrm{MZO}$ buffer deposited at $180 \mathrm{~W}$ and $1 \% \mathrm{O}_{2}$ concentration in the working gas. The efficiency of the completed device was tested immediately after fabrication and then retested after the device was left resting in a sample-box exposed to the atmosphere for 30 days. The device efficiency decreased by approximately $4 \%$ absolute during this timeperiod. The JV curves and performance parameters of a newly fabricated solar cell sample are compared to the performance of the same solar cell after 30 days of ageing in Fig. 2 . The Fill Factor (FF) is the performance parameter which was most affected. Similar devices fabricated with a CdS buffer layer do not degrade. The degradation mechanism is related to the incorporation of the $\mathrm{MZO}$ as the buffer layer. In an attempt to isolate the mechanism involved, a thorough characterisation of the film was carried out comparing the MZO properties as the film is newly deposited and after ageing in the environment. 


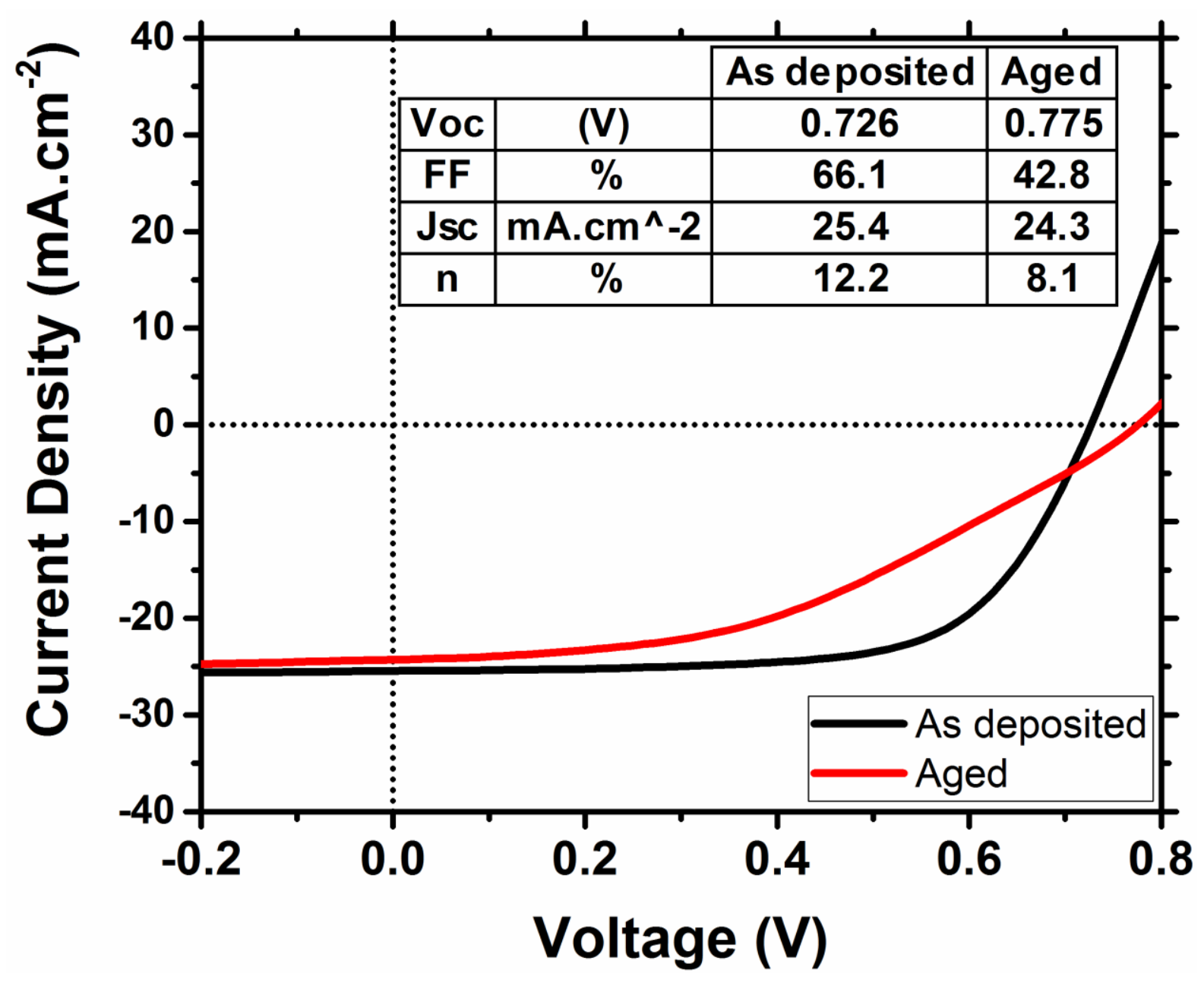

Fig. 2: The comparison between the JV characteristics of an $\mathrm{FTO} / \mathrm{MZO} / \mathrm{CdTe} / \mathrm{Au}$ thin film CdTe solar cell asdeposited and after 30 days of atmospheric exposure.

\subsection{Optical characterisation}

The optical properties of as-deposited samples and aged MZO thin films have been compared. The analysis was carried out on sections of the same thin film, to ensure that the results are comparable. Fig. 3 shows very similar transmittance spectra obtained from an asdeposited and aged MZO thin film. The absorption edge found at short wavelengths is also overlapping, indicating that the semiconductor energy band gap is not varying. These results do not indicate any connection between the bulk optical properties and the drop of device current density output after ageing. 


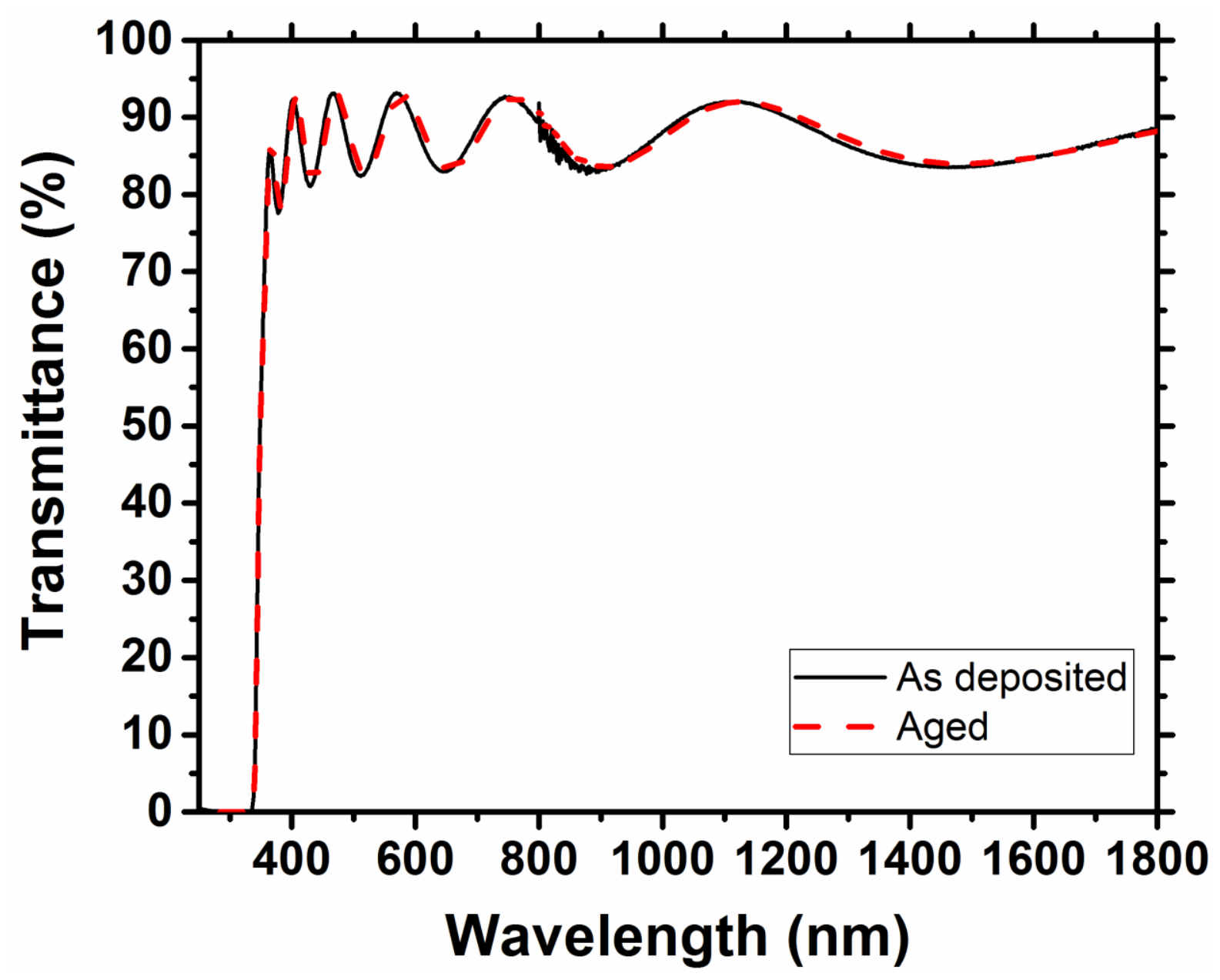

Fig. 3: The optical transmittance spectrum of a $200 \mathrm{~nm}$ thick MZO film as-deposited compared to the spectrum of the same film after 30 days of atmospheric exposure. No discernible change in transmission is observed.

\subsection{Work function measurement}

The measurement of the surface potential of thin film semiconductors can signal the potential contamination or a change in the chemistry of the material surface [15], [16]. The work function of the MZO thin films was measured in atmosphere and monitored with time to establish whether any significant surface chemical degradation occurred related to the device deterioration. The results are presented in Fig. 4 (a), where the evolution of the work function on an hourly basis is shown for the first day of exposure and Fig. 4 (b), where the work function is tracked on a daily basis for 30 days from the film deposition. The results presented in Fig. 4 (a) highlight a very rapid decrease of the film surface work function occurring in the first 4 hours after the film is exposed to the atmosphere, from $3.6 \mathrm{eV}$ to 2.8 $\mathrm{eV}$. After 4 hours of exposure, the variation in the work function slowly reverses to higher work function values. This second phase continues until the $4^{\text {th }}$ day of exposure (Fig. $\left.4 \mathrm{~b}\right)$ ), 
when the work function stabilises at around $3.4 \mathrm{eV}$. The work function was then remeasured after 10 and 30 days of atmospheric exposure and was confirmed to remain at 3.4 $\mathrm{eV}$. These results show that changes to the surface chemistry of the MZO films occurs immediately following exposure to the atmosphere and within the first 5 days of exposure. The changes to the surface chemistry caused by atmospheric exposure initiate as soon as the thin film is removed from vacuum. The effect is most pronounced during the first 4 hours of exposure.

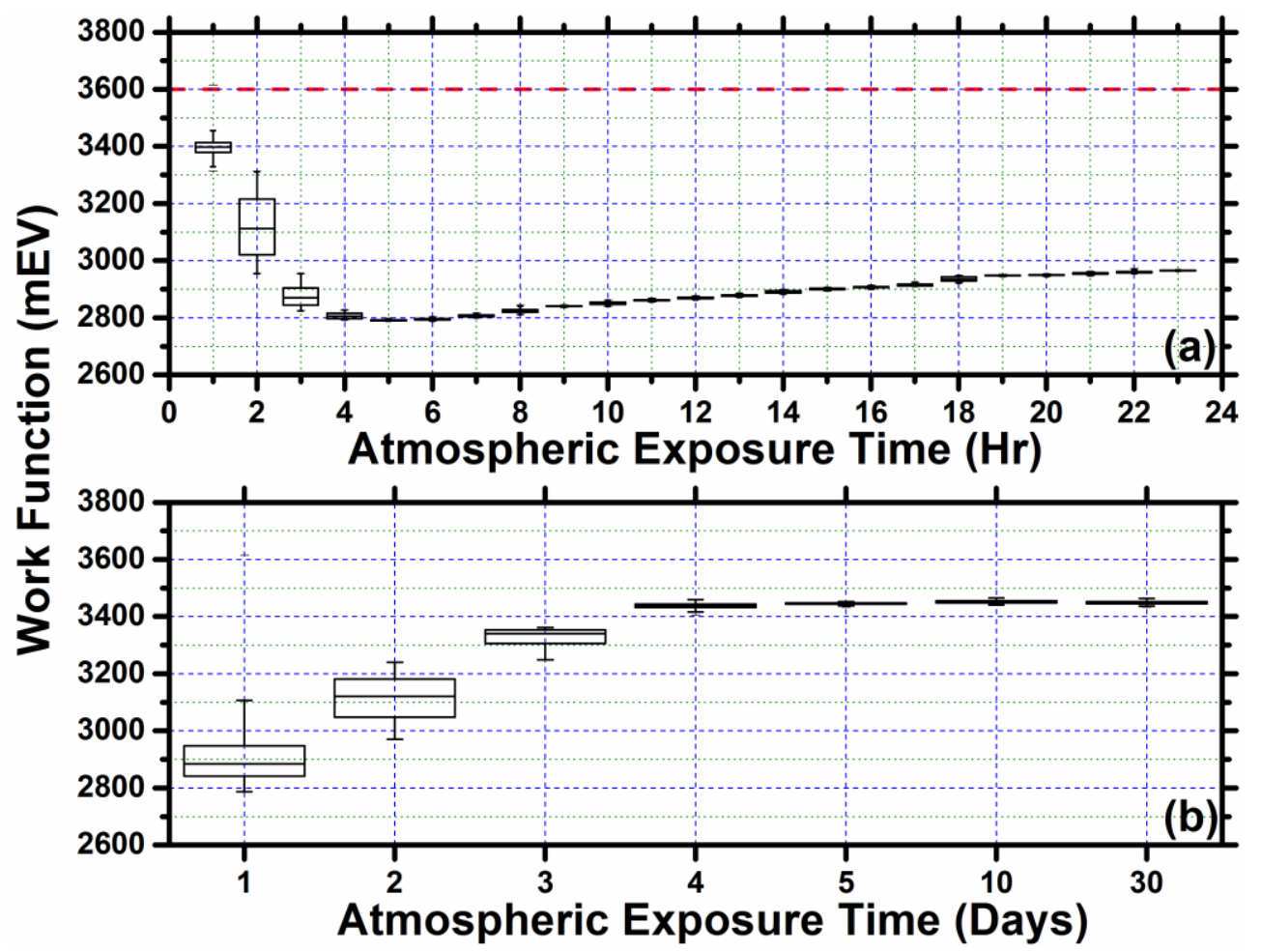

Fig. 4: The analysis of the work function of MZO films highlighting the variation of the surface potential caused by the sample exposure to the atmosphere. In figure (4a) the red dashed line corresponds to the film work function for an as-deposited MZO film, while the box plots show its hourly variation within the first day of exposure. In figure $(4 b$,$) the boxplots illustrate$ the work function variation on a daily basis.

\subsection{XPS analysis of MZO films}

Surface analysis was carried out using X-ray Photoelectron Spectroscopy (XPS) to determine the changes in thin film composition with exposure to the atmosphere. Analysis of the highresolution scan of the $\mathrm{Zn} 2 \mathrm{p}$ peak shows that $\mathrm{Zn}$ exists in its oxidised form with binding energy ranging from $1021.8 \mathrm{eV}$ and $1022.5 \mathrm{eV}$. The presence of metallic $\mathrm{Zn}$, however, cannot be excluded as the peak positions of $\mathrm{ZnO}$ and metallic $\mathrm{Zn}$ partially overlap (metallic $\mathrm{Zn}: 1020.8 \mathrm{eV}-1022.1 \mathrm{eV}$ ). The $Z n 2 p_{3 / 2}$ and $Z n 2 p_{1 / 2}$ of the as-deposited and aged films are unchanged, indicating that the $\mathrm{ZnO}$ bonds in the $\mathrm{MZO}$ thin films are not affected even after the 30 days of atmospheric exposure. However, the $\mathrm{MgO}$ is sensitive to atmospheric 
exposure. The 01s spectra presented in Fig. 5 (b) of the as-deposited and aged thin films is formed by a contribution from two peaks. One peak at $530 \mathrm{eV}$ corresponds to the oxidised state of $\mathrm{Zn}$ and a second peak at $531.6 \mathrm{eV}$ comprises of $\mathrm{MgO}, \mathrm{Mg}(\mathrm{OH})_{2}$ and carbonate components. It is evident that there is an increase in the second peak for the aged thin film sample. This increase is related to one or any combination of the following mechanisms: a) the oxidisation of metallic $\mathrm{Mg}$ in the film, b) the formation of a hydroxide layer caused by $\mathrm{Mg}$ absorbing atmospheric water vapour and c) the surface contamination with adventitious organics. A comparison of the Mg1s peaks is presented in Fig. 5 (c) to identify which of these potential mechanisms is dominant. The Mg1s peak can be sub-divided in two subpeaks, one related to the $\mathrm{MgO}$ bond at $1304.1 \mathrm{eV}$ and the second related to the $\mathrm{Mg}(\mathrm{OH})_{2}$ binding energy at $1303.1 \mathrm{eV}$. The spectrum of the as-deposited thin film indicates that both the oxide and hydroxide states are present in the film. This indicates that the absorption of water vapour by the $\mathrm{Mg}$ in the films is rapid and starts as soon as the thin film is removed from the vacuum in the magnetron sputtering system and exposed to air. This is consistent with the results presented in the previous section showing a significant movement of the surface potential of the MZO films starting immediately the film is removed from vacuum. The aged sample shows a shift in the Mg1s peak indicating that the hydroxide formation has increased after one month of atmospheric exposure. Similarly, the Mg2p spectrum of the aged thin film shows a prevailing $\mathrm{Mg}(\mathrm{OH})_{2}$ peak at $49.2 \mathrm{eV}$. The conversion of oxide to hydroxide explains the inconsistent results obtained using MZO as a buffer and confirms previous work showing that $\mathrm{MgO}$ absorbs atmospheric water vapour [17]-[20]. This study shows the same effect when MgO is incorporated as a dopant in Zinc Oxide.
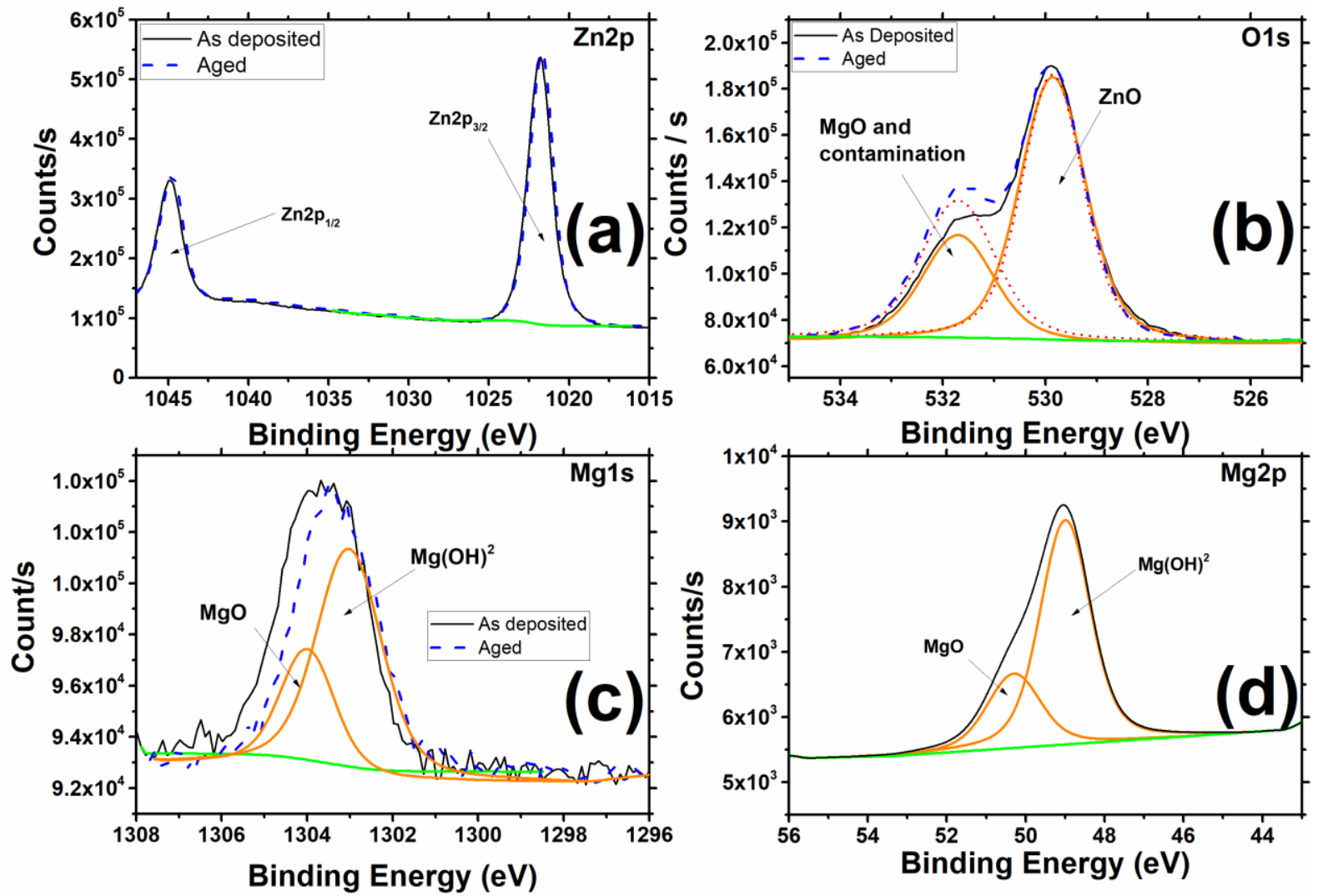
Fig. 5: The results of the XPS analysis carried out on MZO films. Graphs represent the comparison between MZO as-deposited and aged films of the Zn2p peaks (a), of the O1s peaks (b) and of the Mg1s peak (c). Fig. 5 (d) represents only the Mg2p peak relative to the aged sample for clarity. The oxide is converted to hydroxide by exposure to water vapour in the atmosphere.

3.5. The effect of varying sputtering deposition parameters on the stability of MZO films

The results have shown the MZO film is sensitive to the presence of atmospheric humidity. In this section, the effects of varying the deposition conditions on material stability are analysed. The effects of varying the oxygen partial pressure in the working gas and the deposition power have been investigated. The 01s peak has been used as a reference peak to study any significant variation in the material molecular bonding and composition. The results presented in Fig. 6 show that an increased oxygen level affects the film composition and ageing of the film. Figs 6(a), 6(b) and 6(c) show the O1s peak intensities of MZO films deposited at a constant power $(150 \mathrm{~W})$ and increasing $\mathrm{O}_{2}$ concentrations in the working gas atmosphere. The increasing $\mathrm{O}_{2}$ content favours the formation of $\mathrm{MgO}$ and $\mathrm{Mg}$-related compounds, as indicated by the relative increase of the peak at binding energy $532 \mathrm{eV}$ over the peak located at $530 \mathrm{eV}$, which is related to $\mathrm{ZnO}$. The higher the oxygen concentration, the more the material was found to be unstable. The increase of the intensity of the peak related to the $\mathrm{MgO}$ and $\mathrm{Mg}(\mathrm{OH})_{2}$ binding energies compared to the $\mathrm{ZnO}$ peak intensity is significant and becomes even more pronounced for films deposited at higher $\mathrm{O}_{2}$ levels. Figs $6(\mathrm{e}), 6(\mathrm{~b})$ and $6(\mathrm{f})$ show the O1s peak intensities of the MZO films deposited at a constant composition of the working gas and at increasing sputtering power. It is noted that at $120 \mathrm{~W}$ and $180 \mathrm{~W}$ the ZnO-related peak is not significantly affected by the ageing. However, the peak related to $\mathrm{MgO}$ and contamination grows in intensity at both powers. The peak growth, however, is lower than that of MZO films deposited at $150 \mathrm{~W}$. The results of this study show that the sputtering conditions can be used to obtain an MZO thin film less sensitive to atmospheric humidity. However, all the films showed some degradation after 30 days of exposure. The degradation, as shown by the results presented in this and the previous section, are attributed to the absorption of water vapour due to atmospheric humidity by the $\mathrm{MgO}$ present in the film to form $\mathrm{Mg}(\mathrm{OH})_{2}$. 

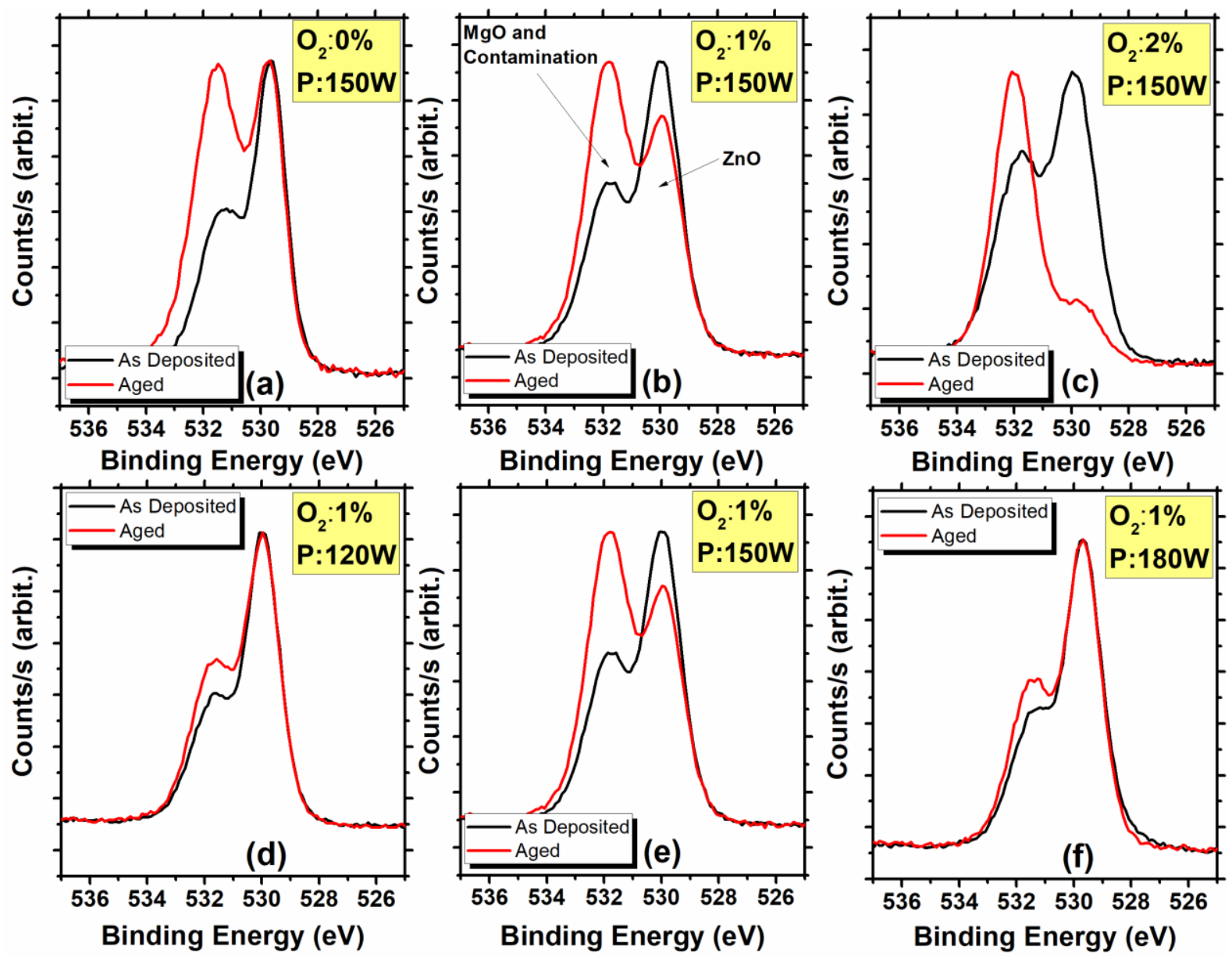

Binding Energy (eV)

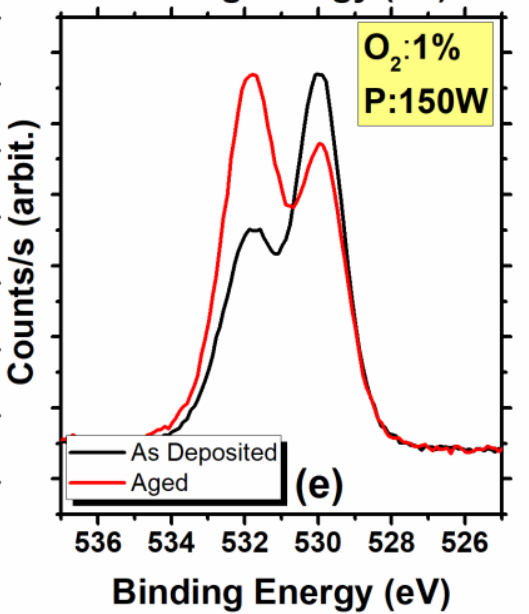

Binding Energy (eV)

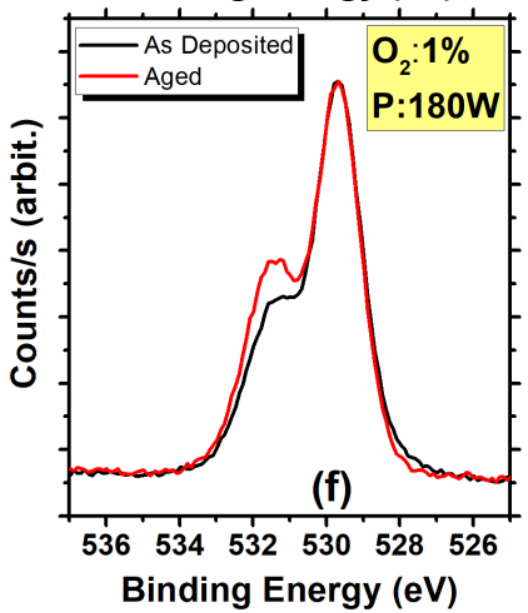

Fig. 6: The effect of film deposition conditions and ageing on the O1s peak. The O1s spectra present two distinctive peaks, one located at $\sim 532 \mathrm{eV}$ and the other one at $\sim 530 \mathrm{eV}$ related to $\mathrm{MgO}$ and $\mathrm{Mg}$-carbonates and $\mathrm{ZnO}$, respectively. The figures show the peaks evolution from as-deposited films (black line) to aged films (red line) for films deposited at different deposition conditions (pressure and oxygen content), which are indicated in the yellow boxes on the top-right corner of each figure.

\section{DISCUSSION}

The results presented on simple thin films of $\mathrm{MZO}$ do not represent exactly the degradation mechanisms occurring in a thin film CdTe photovoltaic device. This is because the MZO thin films have been analysed individually and not within a completed cell, and then exposed directly to atmospheric conditions. It is likely that the changes in surface chemistry will occur at a slower rate when the MZO layer is capped by other semiconductors. Also once the technology is scaled up to module size, an efficient module encapsulation may reduce the degradation rates. Nevertheless, the drop in cell efficiency related the degradation of the MZO is significant and relatively fast. The MgO contained in the MZO films is highly reactive to the water vapour present in humid conditions. The reaction forms a $\mathrm{Mg}(\mathrm{OH})_{2}$ layer which changes the surface chemistry and surface work function. Many studies highlight the importance and stability of the interface chemistry between semiconductors [6], [7], [12], [21]. This study shows that the degradation in the efficiency of CdTe devices 
incorporating an MZO buffer is caused by changes in the interface properties at the $\mathrm{MZO} / \mathrm{CdTe}$ interface and possibly also at the MZO/TCO interface, where secondary barriers can introduce further resistance to the carrier extraction from the solar cell [22], [23].

\section{CONCLUSIONS}

This study has focused on the analysis of degradation occurring in thin film CdTe solar cells incorporating MZO buffer layers. The study aimed to identify the root cause of the degradation. The degradation is not obvious because the changes that occur do not affect the optical properties of the MZO film. However, measurements of the MZO surface potential has shown a rapidly fluctuating surface work function starting immediately the thin film was exposed to the atmosphere and stabilising after 4 days of exposure. The surface work function ranged between $3.6 \mathrm{eV}$ and $2.8 \mathrm{eV}$ and stabilised at $3.4 \mathrm{eV}$. The variation in work function is attributed to changes in the surface composition of the MZO films. XPS analysis shows that the changes that occur to the surface composition are caused by the absorption of water from humidity in the air by the $\mathrm{MgO}$ fraction in the MZO films. The $\mathrm{ZnO}$ is relatively much more stable. Films deposited at different powers and oxygen concentrations were analysed in an attempt to identify deposition conditions that would provide more stable films. The results show that by tuning the deposition conditions it is possible to reduce the film reactivity to atmospheric humidity. However, all the MZO film samples showed degradation. It may be that some post deposition treatment such as rapid thermal annealing could improve stability, but this has not been tested in this study.

Although the use of $\mathrm{MZO}$ results in more efficient solar cells by increasing the short current density Jsc, the degradation is a concern and explains the variation in results from different laboratories around the world. A 25-year warranty is offered with most photovoltaic modules and the degradation in the MZO layer reported in this study means that a more stable transparent buffer layer is required.

\section{ACKNOWLEDGEMENTS}

The Loughborough authors would like to thank support from the EPSRC for funding this research (EP/J017361/1 and EP/N026438/1). The CSU authors are grateful for funding from the National Science Foundation (NSF) Industry/University Collaborative Research Center (I/UCRC) and PFI:AIR-RA program and the U.S. Department of Energy (DOE) Small Innovative Projects in Solar (SIPS).

\section{References}

[1] X. Wu, "High-efficiency polycrystalline CdTe thin-film solar cells," Sol. Energy, vol. 77, no. 6, pp. 803-814, Dec. 2004.

[2] J. M. Kephart, R. Geisthardt, and W. S. Sampath, "Sputtered, oxygenated CdS window layers for higher current in CdS/CdTe thin film solar cells," in 2012 38th IEEE Photovoltaic Specialists Conference, 2012, pp. 000854-000858. 
[3] D. M. Meysing, M. O. Reese, C. W. Warren, A. Abbas, J. M. Burst, H. P. Mahabaduge, W. K. Metzger, J. M. Walls, M. C. Lonergan, T. M. Barnes, and C. A. Wolden, "Evolution of oxygenated cadmium sulfide (CdS:O) during high-temperature CdTe solar cell fabrication," Sol. Energy Mater. Sol. Cells, vol. 157, pp. 276-285, Dec. 2016.

[4] A. Abbas, D. M. Meysing, M. O. Reese, T. M. Barnes, J. M. Walls, and C. A. Wolden, "Structural and chemical evolution of the CdS:O window layer during individual CdTe solar cell processing steps," Sol. Energy, vol. 159, no. December 2017, pp. 940-946, Jan. 2018.

[5] A. Abbas, P. Kaminski, G. West, K. Barth, W. Sampath, J. Bowers, and J. M. Walls, "Cadmium Chloride Assisted Re-Crystallisation of CdTe: The Effect on the CdS Window Layer," MRS Proc., vol. 1738, pp. mrsf14-1738-v03-03, Feb. 2015.

[6] J. M. Kephart, R. M. Geisthardt, Z. Ma, J. McCamy, and W. S. Sampath, "Reduction of window layer optical losses in CdS/CdTe solar cells using a float-line manufacturable HRT layer," in 2013 IEEE 39th Photovoltaic Specialists Conference (PVSC), 2013, pp. 1653-1657.

[7] T. Song, A. Kanevce, and J. R. Sites, "Emitter/absorber interface of CdTe solar cells," J. Appl. Phys., vol. 119, no. 23, p. 233104, Jun. 2016.

[8] F. Bittau, C. Potamialis, M. Togay, A. Abbas, P. J. M. Isherwood, J. W. Bowers, and J. M. Walls, "Analysis and optimisation of the glass/TCO/MZO stack for thin film CdTe solar cells," Sol. Energy Mater. Sol. Cells, vol. 187, no. July, pp. 15-22, Dec. 2018.

[9] A. R. D. Menossi, E. Artegiani, F. Bittau, M. Barbato, M. Meneghini, G. Meneghesso, J.W. Bowers, J.M. Walls, F. Piccinelli, "High Efficiency CdTe Solar Cells by Low Temperature Deposition with MgZnO HRT Layer," in 33rd European Photovoltaic Solar Energy Conference and Exhibition, 2017, pp. 1027-1030.

[10] F. Bittau, E. Artegiani, A. Abbas, D. Menossi, A. Romeo, J. W. Bowers, and J. M. Walls, "Magnesium-doped Zinc Oxide as a High Resistance Transparent Layer for thin film CdS / CdTe solar cells," in: 2017 IEEE 44th Photovoltaic Specialist Conference (PVSC), 2017, pp. 752-756.

[11] J. M. Kephart, J. W. McCamy, Z. Ma, A. Ganjoo, F. M. Alamgir, and W. S. Sampath, "Band alignment of front contact layers for high-efficiency CdTe solar cells," Sol. Energy Mater. Sol. Cells, vol. 157, pp. 266-275, Dec. 2016.

[12] S. Shimakawa, Y. Hashimoto, S. Hayashi, T. Satoh, and T. Negami, "Annealing effects on $\mathrm{Zn1-xMgxO/CIGS} \mathrm{interfaces} \mathrm{characterized} \mathrm{by} \mathrm{ultraviolet} \mathrm{light} \mathrm{excited} \mathrm{time-}$ resolved photoluminescence," Sol. Energy Mater. Sol. Cells, vol. 92, no. 9, pp. 10861090, Sep. 2008.

[13] J. V. Li, X. Li, Y. Yan, C.-S. Jiang, W. K. Metzger, I. L. Repins, M. A. Contreras, and D. H. Levi, "Influence of sputtering a ZnMgO window layer on the interface and bulk properties of $\mathrm{Cu}(\mathrm{In}, \mathrm{Ga}) \mathrm{Se}[\mathrm{sub} 2]$ solar cells," J. Vac. Sci. Technol. B Microelectron. Nanom. Struct., vol. 27, no. 6, p. 2384, 2009.

[14] A. H. Munshi, J. Kephart, A. Abbas, J. Raguse, J. Beaudry, K. Barth, J. Sites, J. Walls, 
and W. Sampath, "Polycrystalline CdSeTe/CdTe Absorber Cells With $28 \mathrm{~mA} / \mathrm{cm} 2$ Short-Circuit Current," IEEE J. Photovoltaics, vol. 8, no. 1, pp. 310-314, 2018.

[15] J. M. Heras and L. Viscido, "Work function changes upon water contamination of metal surfaces," Appl. Surf. Sci., vol. 4, no. 2, pp. 238-241, Mar. 1980.

[16] K. Sugiyama, H. Ishii, Y. Ouchi, and K. Seki, "Dependence of indium-tin-oxide work function on surface cleaning method as studied by ultraviolet and $\mathrm{x}$-ray photoemission spectroscopies," J. Appl. Phys., vol. 87, no. 1, pp. 295-298, Jan. 2000.

[17] M. P. Delplancke-Ogletree, M. Ye, R. Winand, J. F. de Marneffe, and R. Deltour, "Influences of thermal annealing and humidity exposure on surface structure of (100) single-crystal MgO substrate," J. Mater. Res., vol. 14, no. 05, pp. 2133-2137, May 1999.

[18] A. Chowdhury and J. Kumar, "Morphology, surface topography and optical studies on electron beam evaporated MgO thin films," Bull. Mater. Sci., vol. 29, no. 5, pp. 513521, Oct. 2006.

[19] M. Liu, S. Zanna, H. Ardelean, I. Frateur, P. Schmutz, G. Song, A. Atrens, and P. Marcus, "A first quantitative XPS study of the surface films formed, by exposure to water, on Mg and on the Mg-Al intermetallics: Al3Mg2 and Mg17Al12," Corros. Sci., vol. 51, no. 5, pp. 1115-1127, May 2009.

[20] H. . Yao, Y. Li, and A. T. . Wee, "An XPS investigation of the oxidation/corrosion of melt-spun Mg," Appl. Surf. Sci., vol. 158, no. 1-2, pp. 112-119, May 2000.

[21] A. Klein, C. Körber, A. Wachau, F. Säuberlich, Y. Gassenbauer, S. P. Harvey, D. E. Proffit, and T. O. Mason, "Transparent Conducting Oxides for Photovoltaics: Manipulation of Fermi Level, Work Function and Energy Band Alignment," Materials (Basel)., vol. 3, no. 11, pp. 4892-4914, Nov. 2010.

[22] T. Song, J. Tyler McGoffin, and J. R. Sites, "Interface-Barrier-Induced J-V Distortion of CIGS Cells With Sputtered-Deposited Zn(S,O) Window Layers," IEEE J. Photovoltaics, vol. 4, no. 3, pp. 942-947, May 2014.

[23] A. O. Pudov, A. Kanevce, H. a. Al-Thani, J. R. Sites, and F. S. Hasoon, "Secondary barriers in CdS-Culn1-xGaxSe2 solar cells," J. Appl. Phys., vol. 97, no. 6, p. 064901, Mar. 2005. 


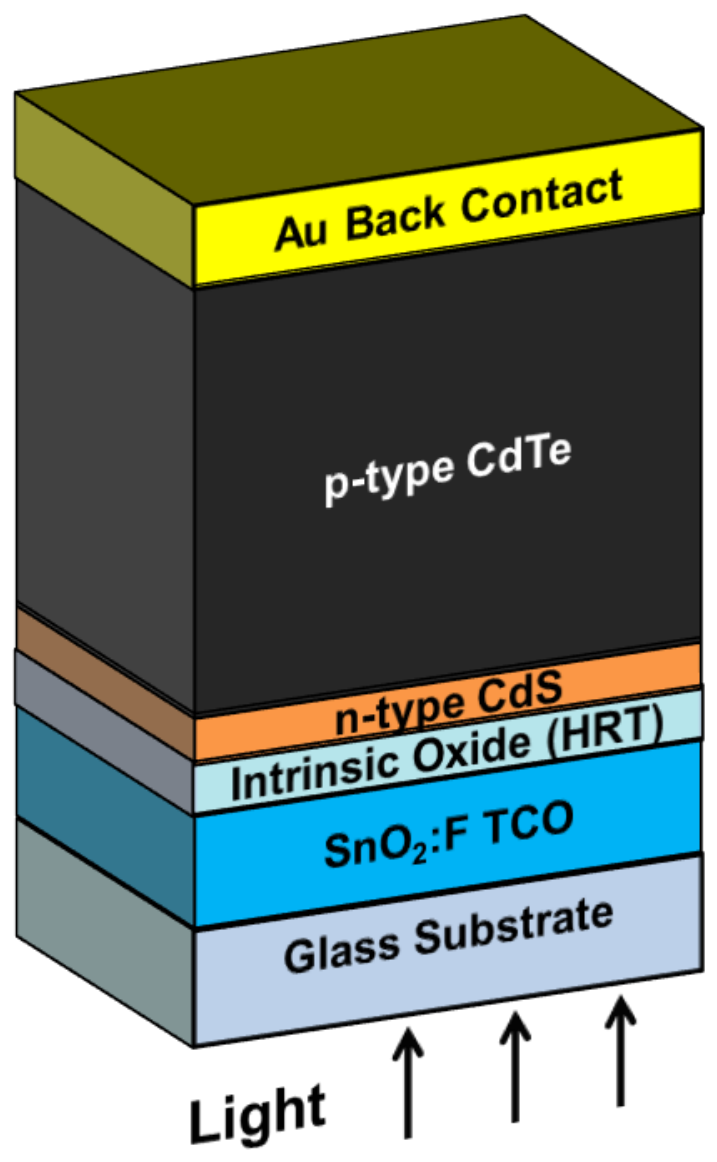

Fig. 1

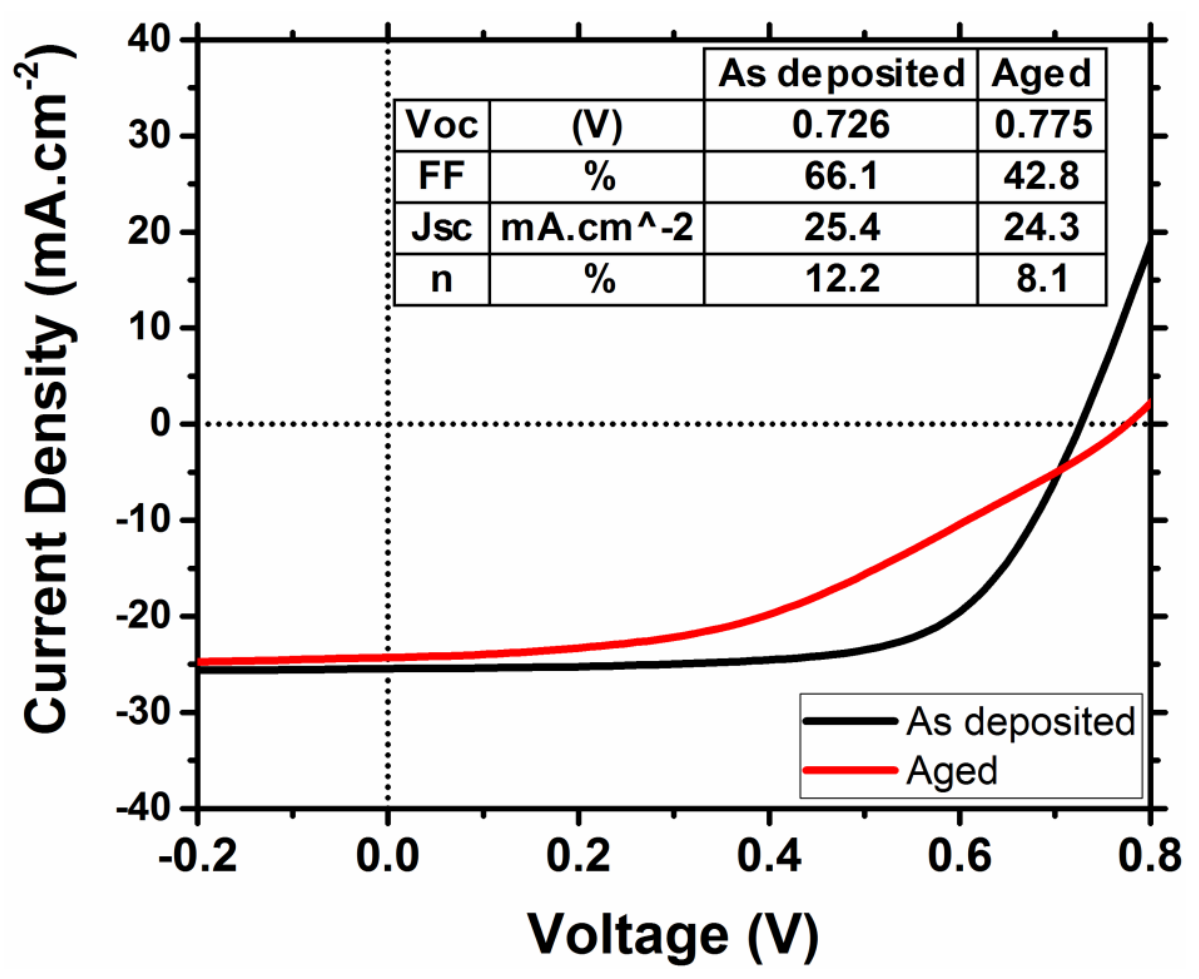

Fig.2 


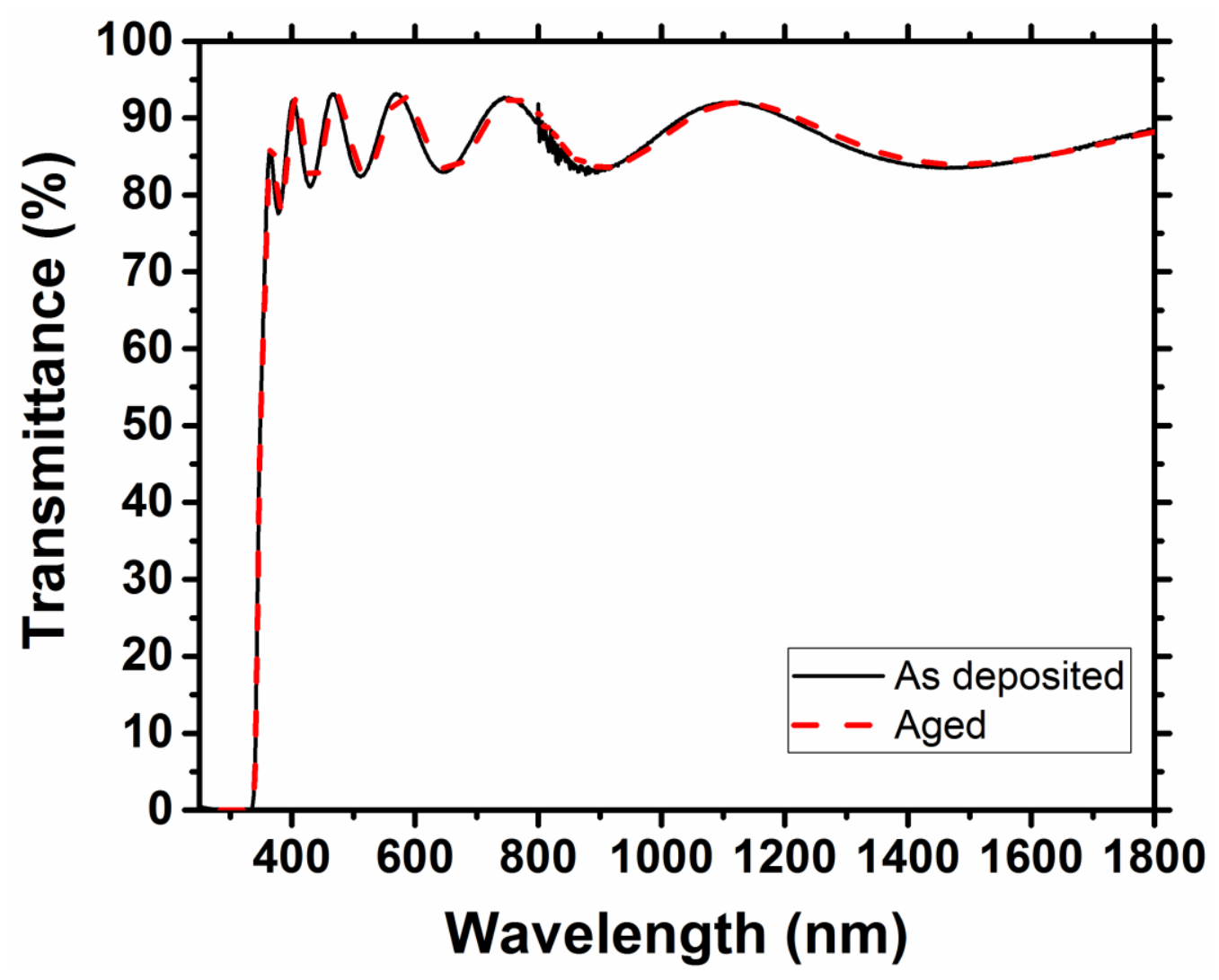

Fig. 3

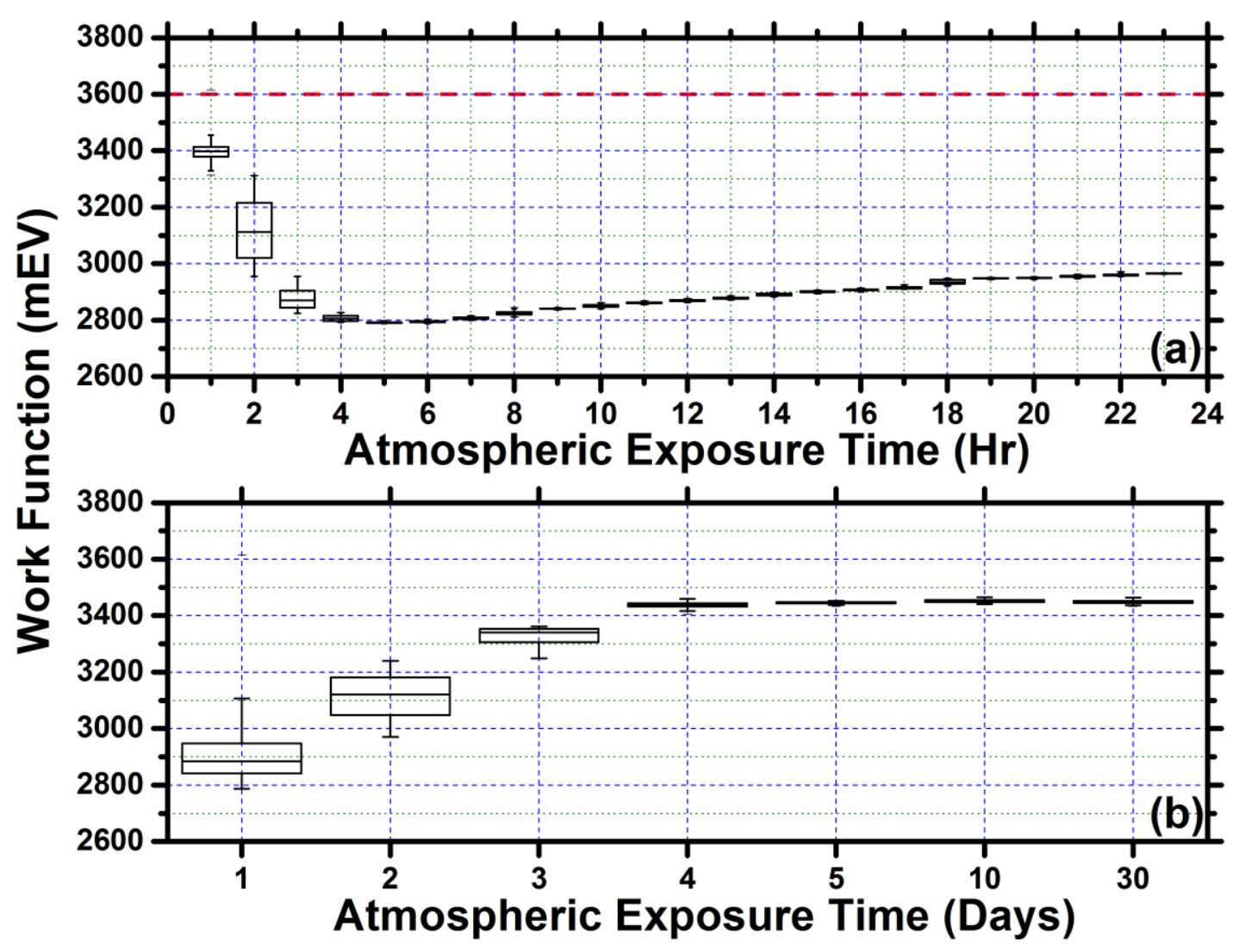

Fig.4 

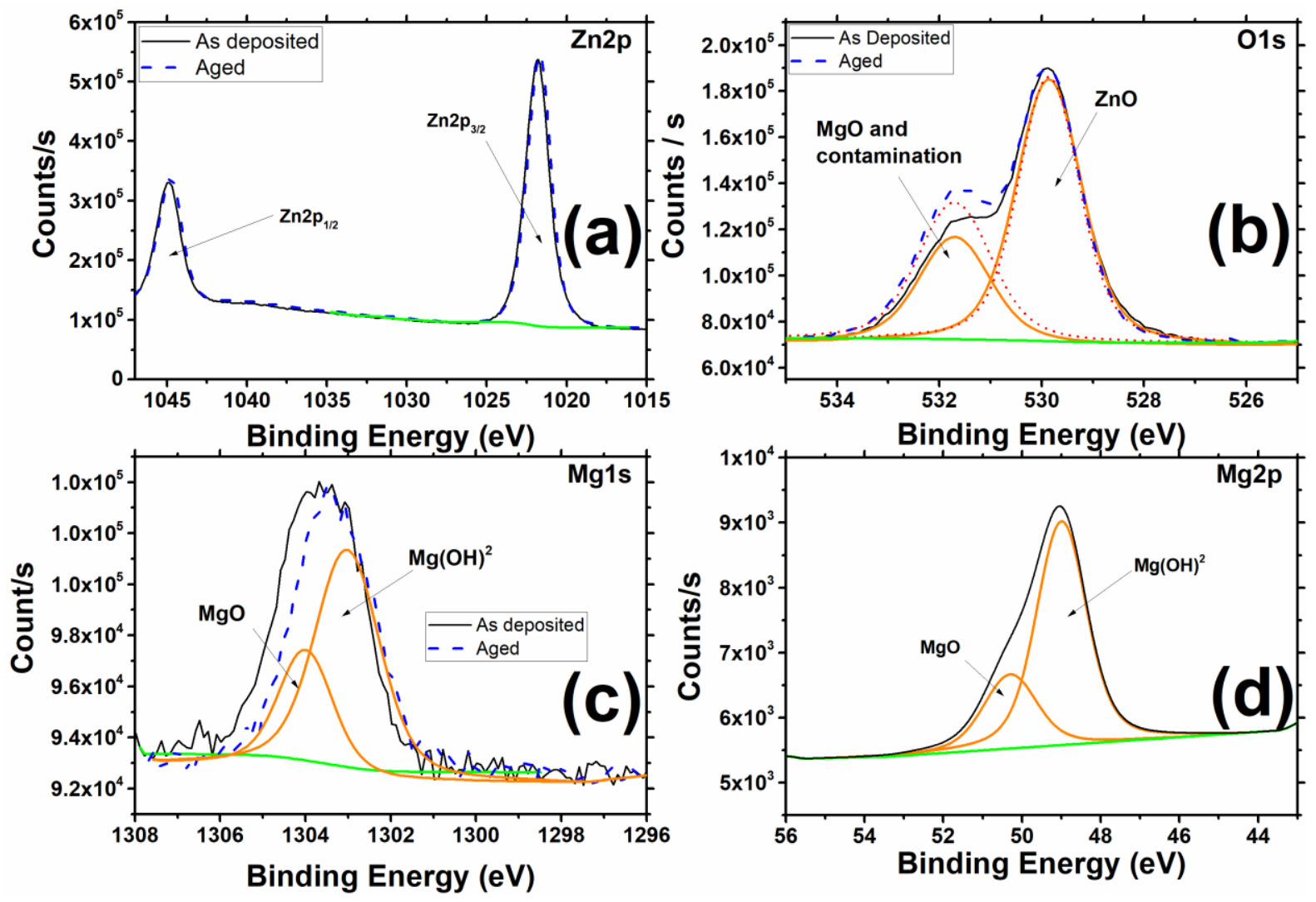

Fig.5

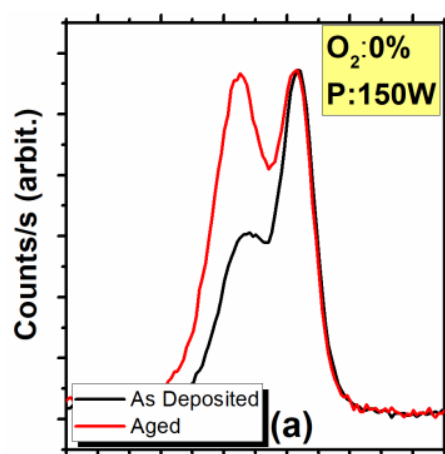

$\begin{array}{llllll}536 & 534 & 532 & 530 & 528 & 526\end{array}$ Binding Energy (eV)

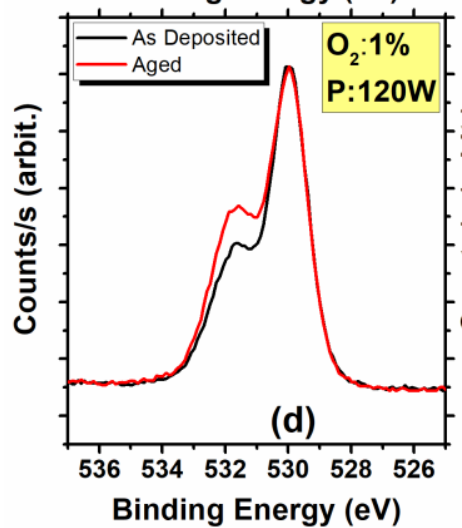

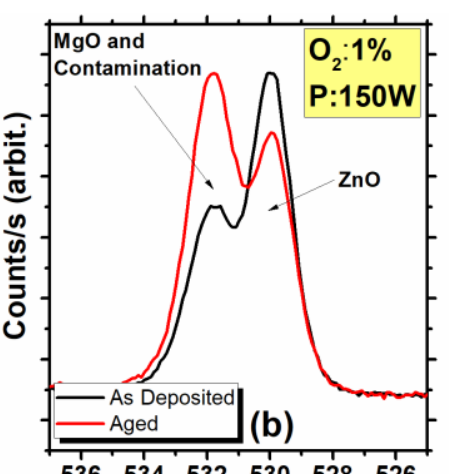

$\begin{array}{lllllll}536 & 534 & 532 & 530 & 528 & 526\end{array}$ Binding Energy (eV)

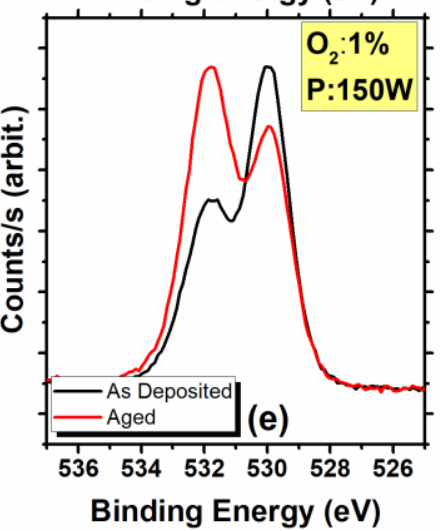

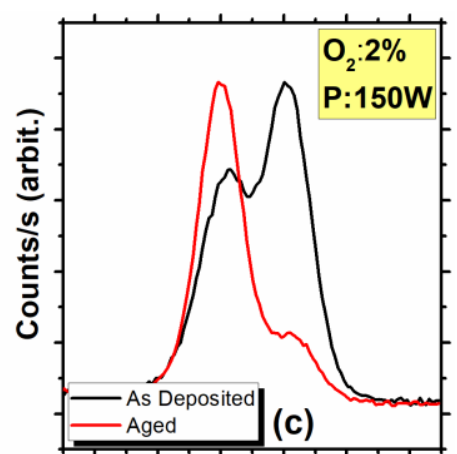

$\begin{array}{lllllll}536 & 534 & 532 & 530 & 528 & 526\end{array}$ Binding Energy (eV)

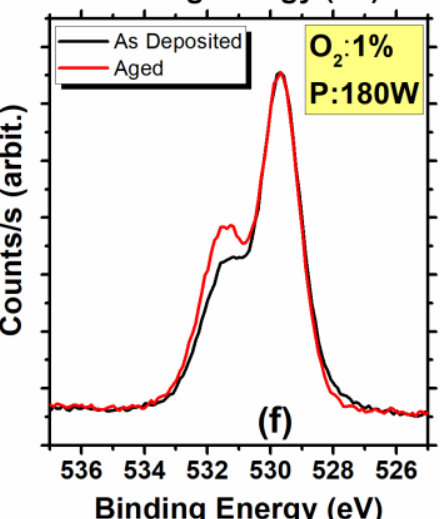

Fig. 6 


\section{CAPTIONS}

Fig. 7: Schematic diagram of the conventional structure of a thin film CdTe solar device. The use of $\mathrm{CdS}$ causes parasitic absorption losses which can be eliminated by using a wide band gap buffer layer such as magnesium doped zinc oxide (MZO).

Fig. 8: The comparison between the JV characteristics of an FTO/MZO/CdTe/Au thin film CdTe solar cell as-deposited and after 30 days of atmospheric exposure.

Fig. 9: The optical transmittance spectrum of a $200 \mathrm{~nm}$ thick MZO film as-deposited compared to the spectrum of the same film after 30 days of atmospheric exposure. No discernible change in transmission is observed.

Fig. 10: The analysis of the work function of MZO films highlighting the variation of the surface potential caused by the sample exposure to the atmosphere. In figure (4a) the red dashed line corresponds to the film work function for an as-deposited MZO film, while the box plots show its hourly variation within the first day of exposure. In figure $(4 b$,$) the$ boxplots illustrate the work function variation on a daily basis.

Fig. 11: The results of the XPS analysis carried out on MZO films. Graphs represent the comparison between MZO as-deposited and aged films of the Zn2p peaks (a), of the O1s peaks (b) and of the Mg1s peak. Fig. 5 (b) represents only the Mg2p peak relative to the aged sample for clarity. The oxide is converted to hydroxide by exposure to water vapour in the atmosphere.

Fig. 12: The effect of film deposition conditions and ageing on the O1s peak. The O1s spectra present two distinctive peaks, one located at $\sim 532 \mathrm{eV}$ and the other one at $\sim 530 \mathrm{eV}$ related to $\mathrm{MgO}$ and $\mathrm{Mg}$-carbonates and $\mathrm{ZnO}$, respectively. The figures show the peaks evolution from as-deposited films (black line) to aged films (red line) for films deposited at different deposition conditions (pressure and oxygen content), which are indicated in the yellow boxes on the top-right corner of each figure. 